\title{
FAST SUMMATION OF DOUBLE INFINITE MODAL SERIES INVOLVED IN ANALYSIS OF SHIELDED MICROSTRIP CIRCUITS
}

\author{
PIOTR M. SŁOBODZIAN \\ Institute of Telecommunications and Acoustics, Wrockaw University of Technology Wyb. \\ Wyspiańskiego 27, 50-370 Wrocław, Poland \\ e-mail: Piotr.Slobodzian@pwr.wroc.pl
}

(Rec. 14 November 2003)

\begin{abstract}
The paper addresses some aspects connected with computational methods involved in analysis of shielded microstrip circuits in the frame of the IE-MoM approach. The paper is focused on a method for efficient evaluation of double infinite modal series, which arise in the analysis of vertical metallizations embedded in a waveguide or cavity filled with a multilayer medium. Generally, the modal series converge very slowly, when treated in its original form, and from practical point of view it makes the IE-MoM approach inefficient. The rate of convergence of the modal series can be significantly increased by means of a transformation of the double infinite series into a fast converging single series. The transformation exploits the contour integral and the residue theorem method in conjunction with the well known Kummer's transformation. The proposed method proved to be very efficient since it enables radical savings in computational time and this feature makes the method a good candidate for practical purposes, especially for electromagnetic CAD tools.
\end{abstract}

\section{INTRODUCTION}

One of a method for rigorous analysis of shielded microstrip circuits is the technique of integral equations (IE) formulated for electric and magnetic fields (or potentials) and their sources distributed over conducting surfaces. The integral equations are usually solved by means of the Method of Moments (MoM) and most frequently by one of its variant, namely the Galerkin's scheme. While the technique for analysis of microstrip circuits consisting of horizontal (planar) metallizations is already well established (see, e.g. [1] and [2]) and applied in commercial CAD software (e.g. in CAD tools offered by Sonnet Software, Inc.) the effective analysis of microstrip circuits containing vertical metallizations still pose a problem. The definition of horizontal and vertical metallizations embedded in a cavity or waveguide filled with a layered medium follows strictly the configuration depicted in Fig. 1.

The question of analysis of vertical metallizations by means of the IE-MoM technique has been discussed recently in [3] and [4]. Although the proposed approach gives us a possibility for inclusion of vertical metallizations in the full-wave analysis of shielded microstrip circuits, its efficient use is limited due to very slow convergence of the involved double infinite modal series. The effectiveness of the analysis depends mainly on the computational effort that is 


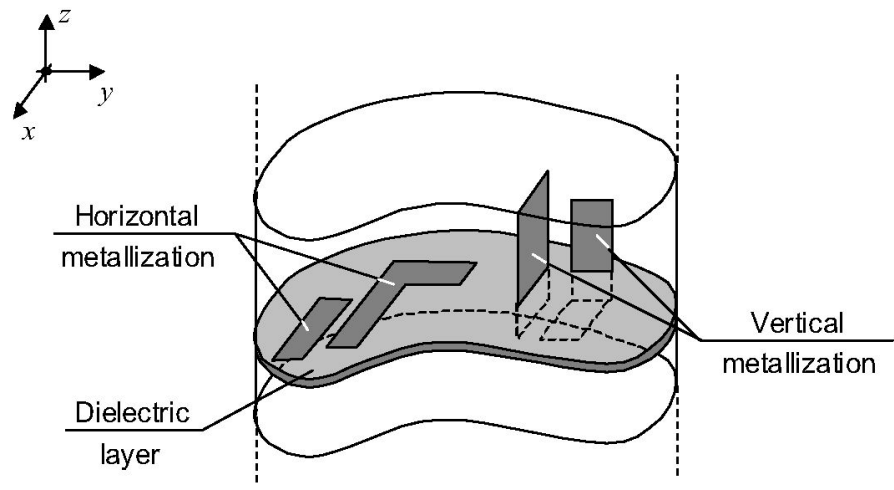

Fig. 1. Uniform waveguide (or cavity) with horizontal and vertical metallizations

made in order to evaluate double infinite modal series describing each entry of the MoM matrix. Numerical treatment of vertical metallizations deserves special attention, since convergence of the resulting series is much more slower than that we need to cope in the case of horizontal metallizations [3]. In this contribution, we shall show that application of the contour integral and the residue theorem method in conjunction with Kummer's transformation allows the double infinite series to be evaluated very rapidly. The proposed approach is based on the analytical transformation of the double infinite series into a single one, which exhibits a very high rate of convergence. In order to explain the idea which stands behind the transformation we need, first, to describe the series under consideration and this question will be addressed in the next section.

\section{MODAL SERIES ASSOCIATED WITH VERTICAL METALLIZATIONS}

The general form of modal series arising in analysis of shielded vertical metallizations does not enable any deeper insight into the nature of their rate of convergence. In order to enable any analytical treatment of the series we need to know their algebraic form and this is possible only when focusing on a case study, for instance a vertical strip embedded in a rectangular cavity. Such a choice is justified from practical point of view since the rectangular shielding is most frequently used in practice. Additionally, if we assume that the vertical strip lays in the yz-plane and the rooftop basis and testing functions, defined on uniform rectangular cells, are applied for MoM analysis (see Fig. 2) then the crucial part of the modal series, which determines the rate of convergence of the whole series takes the following form

$$
Z_{p \delta}=-\frac{2}{a b \cdot \Delta_{z}^{2} \cdot 2 \pi f \cdot \varepsilon} \cdot\left(Z_{1}+Z_{2}+Z_{3}+Z_{4}\right),
$$

where 


$$
\begin{gathered}
Z_{1}=j \cdot \frac{4}{3} \Delta_{z}^{3} k_{0}^{2} \cdot \sum_{m=1}^{\infty} \sum_{n=1}^{\infty} \frac{W_{m n}}{k_{y_{n}}^{2}} \cdot \frac{1}{\beta_{m n}^{2}} \\
Z_{2}=j \cdot 4 \Delta_{z} \cdot \sum_{m=1}^{\infty} \sum_{n=1}^{\infty} \frac{W_{m n}}{k_{y_{n}}^{2}} \cdot \frac{k_{\rho_{m n}}^{2}}{\beta_{m n}^{4}}, \\
Z_{3}=-4 \cdot \sum_{m=1}^{\infty} \sum_{n=1}^{\infty} \frac{W_{m n}}{k_{y_{n}}^{2}} \cdot \frac{k_{\rho_{m n}}^{2}}{\beta_{m n}^{5}}, \\
Z_{4}=4 \cdot \sum_{m=1}^{\infty} \sum_{n=1}^{\infty} \frac{W_{m n}}{k_{y_{n}}^{2}} \cdot \frac{k_{\rho_{m n}}^{2}}{\beta_{m n}^{5}} \cdot\left[2-\cos \left(\beta_{m n} \Delta_{z}\right)\right] \cdot e^{-j \cdot \beta_{m n} \Delta},
\end{gathered}
$$

and where

$$
\begin{gathered}
W_{m n}=\sin ^{2}\left(k_{x_{m}} x_{0}\right) \cdot\left[\cos \left(k_{y_{n}} y_{1}\right)-\cos \left(k_{y_{n}} y_{2}\right)\right]^{2}, \\
\beta_{m n}=\sqrt{k_{0}^{2}-k_{\rho_{m n}}^{2}} \text { and } k_{\rho_{m n}}^{2}=k_{x_{m}}^{2}+k_{y_{n}}^{2}, \\
k_{x_{m}}=\frac{m \pi}{a} \text { and } k_{y_{n}}=\frac{n \pi}{b} .
\end{gathered}
$$

Fig. 2. The roof-top basis function on a vertical strip embedded in a rectangular cavity

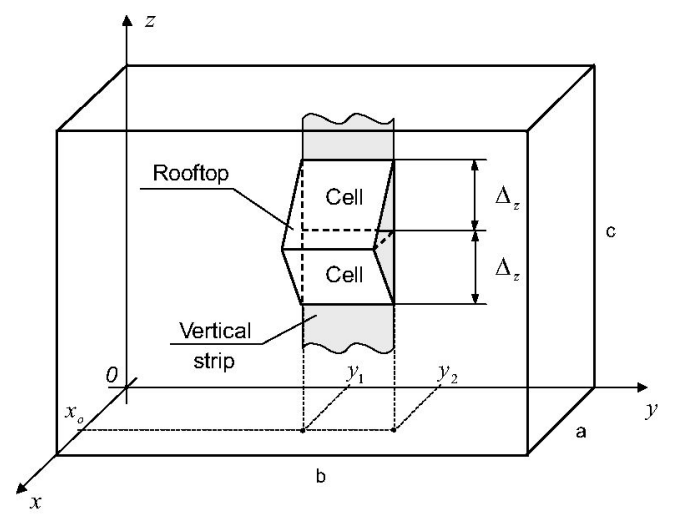

All quantities in Eqs. (2) and (3), which describe the geometry of the problem, i.e. $a, b, x_{0}, y_{1}$, $y_{2}$ and $\Delta_{z}$, are shown in Fig. 2. In addition to this, $k_{0}=2 \pi f \sqrt{\mu \varepsilon}$ in Eq. (3b) is the wave number of the cavity medium and $f$ stands for the frequency at which the microstrip structure is analyzed. It is also important to mention that Eq. (1) describes the modal series associated with a diagonal entry of the MoM matrix, which, generally, requires the highest computational effort during the MoM matrix evaluation. 
Having determined the explicit form of the modal series we are in a position to investigate their convergence. It can be shown that all the series given in Eqs. (2a)-(2d) converge [5], so now we shell focus only on their rate of convergence. In order to do this we shell determine a relative error of the series sum, which precisely reflects the rate of convergence and which can be defined as

$$
\operatorname{Err}(M, N)=\left|\frac{Z_{0}(M, N)-Z_{\text {exact }}}{Z_{\text {exact }}}\right|
$$

where

$$
\begin{aligned}
& Z_{0}(M, N)=\left|\sum_{m=1}^{M} \sum_{n=1}^{N}(\ldots)\right|, \\
& Z_{\text {exact }}=\left|Z_{0}\left(M_{\max }, N_{\max }\right)\right| .
\end{aligned}
$$

In practice, i.e. during the sum evaluation, an infinite double summation is approximated by a finite double summation and this is justified by the fact that in the limit (i.e. when $M_{\max } \rightarrow \infty$ and $\left.N_{\max } \rightarrow \infty\right) Z_{\text {exact }}$ converge to the exact value of the sum (to the limit of the series). Our further analysis will start from comparison between the rate of convergence of the component series $Z_{1}, Z_{2}, Z_{3}$ and $Z_{4}$. All computations have been curried out upon the following assumptions: $a \times b \times c=30 \times 20 \times 10 \mathrm{~mm}, x_{0}=10 \mathrm{~mm}, y_{1}=4 \mathrm{~mm}, y_{2}=6 \mathrm{~mm}$, $\Delta_{z}=2.2 \mathrm{~mm}, f=5.5 \mathrm{GHz}$ and $\left(M_{\max }, N_{\max }\right)=(500,500)$. Additionally, it has been assumed that the cavity is filled with a linear, homogenous and isotropic medium with the intrinsic parameters equal to that of free-space $\left(\varepsilon=\varepsilon_{0}\right.$ and $\left.\mu=\mu_{0}\right)$. Furthermore, the relative error of the sum of the modal series associated with a diagonal entry of the MoM matrix strictly reflects the relative error of circuit parameters of the analyzed structure (e.g. the relative error of its $S$-parameters). Consequently, it has been also assumed that the sufficient level of the relative error, which allows the computations to be accepted as accurate, should take values below $10^{-3}$. The computations has been curried out using the direct summation method for a square set of series terms, i.e. it has been assumed that $M=N$, so the total number of summation is $\mathrm{M} \times \mathrm{N}=M^{2}=N^{2}$. The results of calculation, shown in Fig. 3, reveal that in order to obtain the required level of the relative error for the sum of $Z_{3}$ and $Z_{4}$ we need to take about $2 \cdot 10^{3}$ first terms in the final calculation. On the other hand, series $Z_{1}$ and $Z_{2}$ exhibit an extremely slow rate of convergence and a huge number of terms $\left(>40 \cdot 10^{3}\right)$ must be taken into account during the final calculation in order to reach the required level of the error. This fact makes the analysis highly inefficient and restricts its use for practical applications.

Nevertheless, it is possible to enhance the efficiency of $Z_{1}$ and $Z_{2}$ summation by means of amethod that allows the convergence of the series to be greatly accelerated. In this contribution we shall show that Kummer's transformation in conjunction with the contour 
integral and the residue theorem method enables extremely efficient series evaluation and this question will be addressed in detail in the next section.

\section{SERIES TRANSFORMATION}

The proposed method for series transformation consist of two stages. Firstly, we shall transform the sum of $Z_{1}$ and $Z_{2}$ using Kummer's method in order to separate the static and dynamic part of the resulting series. Secondly, we shall transform these both parts (i.e. the resulting double infinite series) into single infinite series, what drastically reduces the required computational effort.

The concept of Kummer's transformation is discussed in great detail in [6]. Upon applying this transformation to the sum of $Z_{1}$ and $Z_{2}$, provided that their static part is determined for $f \rightarrow 0$, we can write

$$
Z_{1}+Z_{2}=Z_{12}^{\text {stat }}+Z_{12}^{\text {dyn }}
$$

where

$$
\begin{gathered}
Z_{12}^{\text {stat }}=j \cdot 4 \Delta_{z} \cdot\left(1-\frac{1}{3} \Delta_{z}^{3} k_{0}^{2}\right) \cdot W_{1}, \\
Z_{12}^{\text {dyn }}=j \cdot \frac{4}{3} \Delta_{z}^{3} k_{0}^{4} \cdot W_{2}+j \cdot 8 \Delta_{z} k_{0}^{2} \cdot W_{3}-j \cdot 4 \Delta_{z} k_{0}^{4} \cdot W_{3},
\end{gathered}
$$

and where

$$
\begin{gathered}
W_{1}=\sum_{m=1}^{\infty} \sum_{n=1}^{\infty} \frac{W_{m n}}{k_{y_{n}}^{2}} \cdot \frac{1}{k_{\rho_{m n}}^{2}}, \\
W_{2}=\sum_{m=1}^{\infty} \sum_{n=1}^{\infty} \frac{W_{m n}}{k_{y_{n}}^{2}} \cdot \frac{1}{k_{\rho_{m n}}^{2} \beta_{m n}^{2}}, \\
W_{3}=\sum_{m=1}^{\infty} \sum_{n=1}^{\infty} \frac{W_{m n}}{k_{y_{n}}^{2}} \cdot \frac{1}{\beta_{m n}^{4}}, \\
W_{4}=\sum_{m=1}^{\infty} \sum_{n=1}^{\infty} \frac{W_{m n}}{k_{y_{n}}^{2}} \cdot \frac{1}{k_{\rho_{m n}}^{2} \beta_{m n}^{4}} .
\end{gathered}
$$

The gain resulting from the use of Kummer's transformation pertains to a reduction in computational effort due to the fact that $Z_{12}^{\text {stat }}$ does not depend on the frequency at which circuit analysis is curried out. Hence, the computations in the frequency loop confine to evaluation of $Z_{12}^{\text {dyn }}$, which is a rapidly converging series, and then to addition of the obtained result to 
the previously evaluated static part of the modal series. It is worth to note that the static part is evaluated only once before the frequency loop is entered.

In the second stage, the computational effort involved in evaluation of all the series described in Eqs. (9a)-(9b), and especially involved in the static part evaluation, can be further reduced via a transformation of a double infinite series into a single infinite series. The transformation consist in application of the contour integral and the residue theorem method. In order to demonstrate the use of the method we shall transform, for instance, the series $W_{2}$. To do this, let us write down the series as follows

$$
W_{2}=\sum_{m=1}^{\infty} \sum_{n=1}^{\infty} F_{n} \cdot G_{m n}=\sum_{n=1}^{\infty}\left(F_{n} \cdot G_{n}\right),
$$

where

$$
\begin{gathered}
F_{n}=\frac{\left[\cos \left(k_{y_{n}} y_{1}\right)-\cos \left(k_{y_{n}} y_{2}\right)\right]^{2}}{k_{y_{n}}^{2}}, \\
G_{n}=\sum_{m=1}^{\infty} G_{m n}=\sum_{m=1}^{\infty} \frac{\sin ^{2}\left(k_{x_{m}} x_{0}\right)}{k_{\rho_{m n}}^{2} \beta_{m n}^{2}}=-\frac{1}{2}\left(\frac{a}{\pi}\right)^{4} \cdot \sum_{m=1}^{\infty} \frac{\sin ^{2}\left(\frac{1}{2} m \alpha\right)}{m^{4}+\beta_{n} m^{2}+\gamma_{n}},
\end{gathered}
$$

and where

$$
\begin{gathered}
\beta_{n}=\left(\frac{a}{\pi}\right)^{2} \cdot\left(2 k_{y_{n}}^{2}-k_{0}^{2}\right), \\
\gamma_{n}=\left(\frac{a}{\pi}\right)^{4} \cdot k_{y_{n}}^{2} \cdot\left(k_{y_{n}}^{2}-k_{0}^{2}\right), \\
\alpha=2 \pi \cdot \frac{x_{0}}{a} .
\end{gathered}
$$

The relation in Eq. (10) is justified since $W_{2}$ is a convergent series [5]. Our further considerations will focus on the infinite series $G_{n}$. This series can be expressed by means of a contour integral of a function of a complex variable $z=x+j \cdot y$ as follows [7]

$$
F(z)=\frac{e^{j \cdot \alpha z}}{P(z)} \cdot \frac{1}{e^{j \cdot 2 \pi z}-1},
$$

where

$$
P(z)=z^{4}+\beta_{n} z^{2}+\gamma_{n}
$$


It is worth mentioning that such a representation is possible because the summand in Eq. (12) is an even function of $m$. Since $F(z)$ is analytical within a region bounded by any simplyconnected closed curve $C_{0}$, except for a finite number of poles, which in our case are generated by zeros of $P(z)$ and $\left(e^{j \cdot 2 \pi z}-1\right)$, then the contour integral of $F(z)$ along $C_{0}$ is entirely determined by the poles of $F(z)$ enclosed by the contour. Furthermore, it can be shown that when the contour $C_{0}$ has a rectangular shape, shown in Fig. $4\left(C_{0}=C_{1}+C_{2}+C_{3}+C_{4}\right)$, and extends to infinity than the contour integral along $C_{0}$ can be expressed, using the residue theorem, as follows

$$
\oint_{C_{0} \rightarrow \infty} F(z) d z=2 \pi j \cdot \sum_{m=-\infty}^{\infty} \operatorname{Res}\left\{F(z), z_{m}=m\right\}+2 \pi j \cdot \sum_{i=1}^{4} \operatorname{Res}\{F(z), \text { at zeros of } P(z)\}=0 .
$$

It is clear from Eq. (16), that the zeros of $P(z)$ are of great importance for the summation of series $G_{n}$. In our case the zeros are determined by

$$
\begin{gathered}
z_{1,2}= \pm j \cdot \frac{a}{b} n \\
z_{3,4}= \pm j \cdot \frac{a}{b} \sqrt{n^{2}-\left(\frac{b}{\pi}\right)^{2} k_{0}^{2}} .
\end{gathered}
$$

When the cavity medium is lossless and $n>2 \cdot b / \lambda_{0}$ ( $\lambda_{0}$ is the free-space wavelength) then all the zeros are purely imaginary and lay on the $y$-axis. Special care must be exercised, when using Eq. (16), in the case of a lossless medium and $n \leq 2 \cdot b / \lambda_{n}$, since in this case zeros $z_{3,4}$ are purely real and can overlap with zeros generated by $\left(e^{j \cdot 2 \pi z}-1\right)$. In general, when the medium is lossy, zeros $z_{3,4}$ are complex and lay off the both axis.

Finally, Eq. (16) allows the sum of series $G_{n}$ to be evaluated in a closed form, and the result takes the following form

$$
G_{n}=-j \frac{\pi}{2} \cdot\left(\frac{a}{\pi}\right)^{4} \cdot \sum_{i=1}^{4} \frac{1}{4 z_{i}^{3}+2 \beta_{n} z_{i}} \cdot \frac{e^{j \cdot \alpha z_{i}}-1}{e^{j \cdot 2 \pi z_{i}}-1}
$$

where $z_{i}$ stands for the $i$-th zero of $P(z)$ defined in Eq. (17). Upon substituting this new form of series $G_{n}$ into Eq. (10) we get a one dimensional counterpart of the double infinite series $W_{2}$, and this step finishes the second stage of the proposed method for series transformation. The transformation can be also applied to the remaining series, i.e. $W_{1}, W_{3}$ and $W_{4}$, what results, finally, in a huge reduction in the required computational effort during the modal series evaluation.

The gain resulted from application of the proposed method is clearly shown in Fig. 5, where we can compare between the relative error of the sum of the component series $Z_{1}$ and $Z_{2}$ obtained for various methods of series summation. The results of calculations have been curried out under conditions that have been already assumed for calculations referred in Fig. 3. 
As we can see, the pure Kummer's transformation is a very powerful tool in summation of the modal series. Using this method it suffice to take about 100 terms from a square set of terms, i.e. $(M, N)=(10,10)$, to get the relative error below $10^{-3}$. Obviously, before we did the summation at a given frequency $f$ we must, first, determine the static part of the series (see, Eq. 7), which converge as slowly as the original series does (it can be easily verified that the rate of convergence of $Z_{12}^{\text {stat }}$ is very close to that of series $Z_{1}$ and $Z_{2}$ ). The results shown in Fig. 5 have been obtained for the static part evaluated using $500 \times 500$ terms. Therefore, the actual gain resulted from the use of the pure Kummer's transformation is measurable when we need to repeat calculations many times in the frequency loop, what is obviously the case in the microstrip circuits analysis.

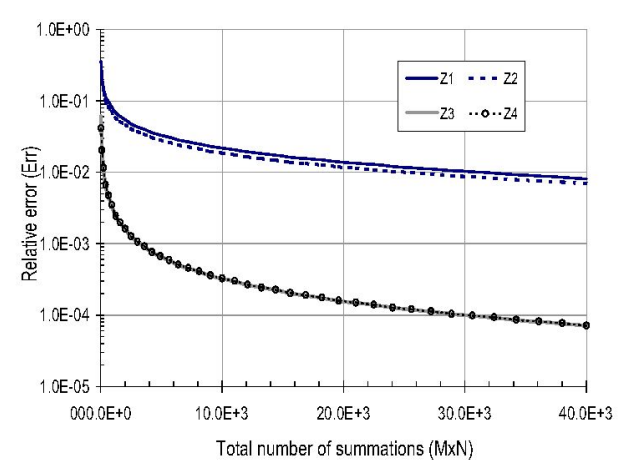

Fig. 3. Relative error of the sum of the component series $Z_{1}, Z_{2}, Z_{3}$ and $Z_{4}$ obtained for the square set of series terms

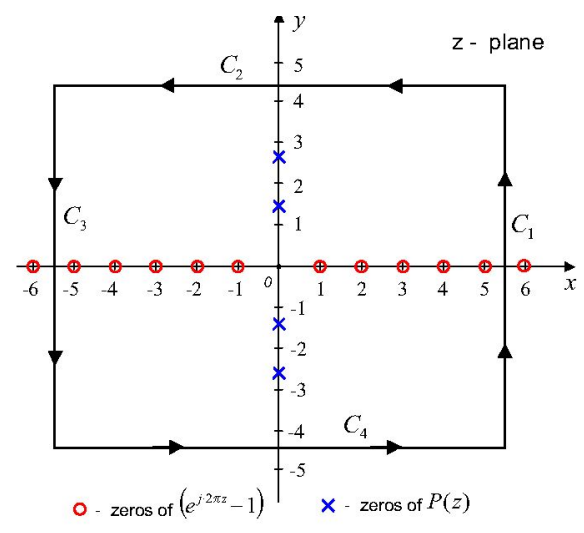

Fig. 4. Contour for evaluation of infinite series $S_{2}$ and position of poles of $F(z)$

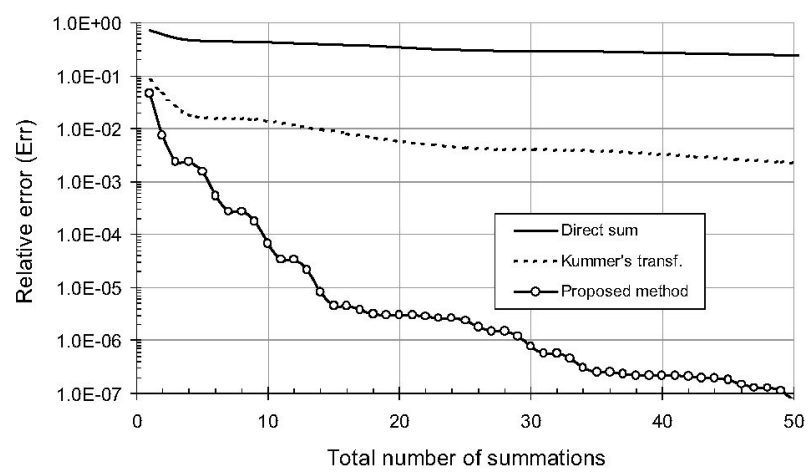

Fig. 5. Relative error of the sum of the component series $Z_{1}, Z_{2}$ obtained for various methods applied to series summation

The computational effort drastically reduces when we combine Kummer's transformation with the double-to-single series transformation (the proposed method). Although in this case we also need to determine the static part of the series, its very precise evaluation requires only 
50-70 first terms of the transformed (single) series. Then, the calculations in the frequency loop can be done very effectively using up to 6-10 first terms of the dynamic part of the series and this enables very effective analysis of microstrip circuits in a wide frequency range.

\section{SUMMARY}

The paper addresses some aspects connected with computational methods involved in analysis of shielded microstrip circuits in the frame of the IE-MoM approach. It has been shows that extremely slowly converging modal series involved in the analysis can be evaluated very effectively by means of Kummer's transformation combined with double-tosingle series transformation. Although, the proposed method proved to work extremely effective its use requires much analytical work, which must be done in advance in order to obtain an algebraic form of the series to be transformed.

Unfortunately, the double-to-single series transformation described in this contribution, can not be directly applied to the series $Z_{3}$ and $Z_{4}$, since generally, due to the definition of

$\beta_{m n}$, they are mulitvalued series. Each summand of these series have two branch points, which are not an isolated singularities, and hence the residue theorem can not be exploited. Furthermore, the contour integral that is involved in the transformation includes an integration along branch cuts and must be evaluated numerically, and this is, in general, a very complicated and time consuming task.

\section{References}

[1] J. C. Rautio and R. F. Harrington, An Electromagnetic Time-Harmonic Analysis of Shielded Microstrip Circuits, IEEE Trans. Microwave Theory Tech., 35, 726-730 (1987).

[2] G. V. Eleftheriades, J. R. Mosig, and M. Guglielmi, A Fast Integral Equation Techniquefor Shielded Planar Circuits Defined on Nonuniform Meshes, IEEE Trans. Microwave Theory Tech., 44, 2293-2296 (1996).

[3] P. M. Słobodzian, A. Alvarez-Melcon, T. M. Grzegorczyk and F. E. Gardiol, Green's function for vertical current sources embedded in uniform waveguides and cavities filled with multilayered media, Microwave Opt. Technol. Lett., 33, 186-191 (2002).

[4] P.M Słobodzian, On the dyadic Green'sfunction in the source region embedded in waveguides or cavitiesfilled with a stratified medium, Microwave Opt. Technol. Lett., 35 93-97 (2002).

[5] V. G. Celidze, Some methodsfor evaluation of double series and double integrals, Tbilisi University Press, Tbilisi, 1977 (inRussian).

[6] K. Knopp, Infinite series, PWN, Warsaw, 1956 (in Polish).

[7] P. M. Morse and H. Feshbach, Methods of theoretical physics, McGraw-Hill Book Co., Inc., New York, 1953. 